\section{Diabetic nephropathy in type 2 diabetes - from pathological mechanisms to clinical occupational medicine practice}

Marina Ruxandra Oțelea', Daniela Dragu

' University of Medicine and Pharmacy "Carol Davila",

Clinical Department 2, Bucharest, Romania

${ }^{2}$ Marie Curie Clinical Hospital, Bucharest, Romania

\section{Corresponding author}

Marina Ruxandra Oțelea

marina.otelea@umfcd.ro

\begin{abstract}
Employees with diabetes and nephropathy need special medical surveillance that involves occupational medicine specialists. However, diabetes is not a unique phenotype and each patient need to be carefully assessed. Age, gender, body mass index, renal function impairment (eGFR, creatinine, urea, uric acid), indicators of diabetes control (fasting glycaemia and $\mathrm{HbA1C}$ ), the presence of co-morbidities, dyslipidaemia, level of serum albumin and total protein, cytokines and other inflammatory markers should be considered in a comprehensive evaluation of the severity of the chronic kidney disease and of the treatment plan. Chronic kidney disease in type 2 diabetes has many facets and various degrees of severity; therefore, permanent communication between the occupational medicine specialist and the treating physician should be maintained. For this purpose, this article reviews the current pathological mechanisms proposed for the explanation of the chronic kidney disease, the diagnostic and the general therapeutic recommendations and also the possible occupational interventions in patients with type 2 diabetic nephropathy.
\end{abstract}

Keywords: type 2 diabetes, chronic kidney disease, occupation

\section{Introduction}

The prevalence of diabetes in Romanian adult population is $11.6 \%$ and of the prediabetes is $16.5 \%$ [1]. The fact that $2.4 \%$ of the Romanian population has undiagnosed diabetes is of major interest for the occupational physician. The patient in this category will honestly declare not having diabetes on the occasion of periodical medical examination. According to the same study, the prevalence of impaired glucose regulation (diabetes and prediabetes) during the active life is $11.5 \%$ in men and $8.1 \%$ in women aged 20-39 years and raises up to $35.5 \%$ in men and to $29.4 \%$ in women of $40-59$ years old. Due to this high prevalence, understanding the characteristics and the factors influencing the evolution of this disease becomes more and more important for the fitness for work assessments and the organisational support recommended by an occupational physician. With a comprehensive and integrated approach covering both social and medical interventions, a patient with diabetes should not develop complications that limit the ability to work. Unfortunately, the current situation is far from the optimal one and the complications are frequent. Acute complications, particularly hypoglycaemia, have direct effects on the fitness capacity and require special individual evaluation in jobs at risk of accidents or in activities needing permanent vigilance. 


\section{Pathogenic mechanisms of chronic kidney disease in type 2 diabetes}

The microangiopathy, clinically expressed by its localization in the retina vessels, in glomeruli and vasa nervorum, is a result of the prolonged exposure to metabolic changes, oxidative stress and advanced glycated products formation. The endothelial dysfunction is illustrated by the high levels of endothelin, a strong pro-inflammatory and vasoconstrictor peptide [2] and by the lost of the capacity of autoregulation in the peripheral vessels. In what concerns the T2D nephropathy pathogenesis, the hyperfiltration is a critical, but potentially manageable element in the early stages of the disease, with the aid of the glycaemic control.

Chronic hyperglycaemia is associated with a hyperosmolar status; it also increases the sodium reabsorption in proximal tubule that reduces the tubuloglomerular feedback, with vasodilatation of the afferent arteriole and decrease of the sodium delivery to the macula; this latter effect stimulates angiotensin II release that constricts the efferent arteriole. Both vasodilatation of the afferent arteriole and constriction of the efferent arteriole lead to hyperperfusion and hyperfiltration, alters the glomerular hemodynamics and causes mechanical strain on the glomerular endothelial and epithelial cells. Hemodynamic stress, high intraglomerular pressure and hyperglycaemia contribute to the release of angiotensin II, cytokines and growth factors (such as transforming growth factor- $\beta$, platelet-derived growth factor- $\beta$ and vascular endothelial growth factor), that orchestrate the mesangial cell matrix expansion and the thickening of the glomerular basal membrane [3]. The high content of glucose alters the normal metabolism and leads to high levels of oxygen reactive species (ROS) formation, another key element in the vascular proliferation, fibrosis and chronic inflammation in the glomeruli and in the interstitium. ROS stimulate the non-enzymatic glycosylation and the advanced glycosylation products formation, activates the polyol and protein kinase $C$ pathway (PKC) and angiotensin II. PKC stimulates transforming growth factor and vascular endothelial growth factor formation, aggravating the hemodynamic deleterious effects.

These interlinked pathological mechanisms were initially described to cause glomerular lesions (the classical glomerulosclerosis, designated also as diabetes nephropathy). But fibrosis and inflammation is not restricted to the glomeruli: tubular atrophy and interstitium fibrosis, together with vascular disease apart from the glomeruli might occur. Therefore, the new concept of "diabetic chronic kidney disease" was proposed to replace the "diabetic nephropathy" [4].

In view of the novel concept of the kidney impact of diabetes, the patients could be divided according to the histological factors in patients with solely glomerular nephropathy (defined by the classical nodular or global glomerulosclerosis or "diabetic podocytopathy") and patients with diabetic CKD with histological lesions that involved, beside the glomerules, the tubules, the interstitium and/or the vessels [5].

\section{The contribution of environmental exposure to type 2 diabetes nephropathy}

Concerning environmental and/or occupational exposure in the pathogeny of diabetes, several studies incriminated persistent organic pollutants (polychlorinatedbiphenyls, organochlorinepesticides, polyciclic aromatic hydrocarbons, phthalates etc) [6], metals (arsenic, cadmium) [7]. Therefore, with proper technical masures, all employees should avoid occupational contact with these substances.

In patients already diagnosed with T2D, nephrotoxic substances (heavy metals, silica, organic solvents and carbon disulphide) contact should be also avoided. Oxidative stress (generation of ROS and reactive nitrogen species, lipid peroxidation and DNA oxidation) seem to be the main toxic effect of arsenic [8] or lead poisoning [9]. Tubular renal cells are the first to be affected in heavy metal chronic exposure, but glomeruli are not turned aside. For example, extensive glomerular fibrotic lesions were observed in experimental exposure to mercury [10].

Chronic exposure to heavy metals leads to Fanconi syndrome (aminoaciduria, glycosuria and loss of ions) [11]. Solvents damage the capillary basement membrane or the tubular epithelia initiating an autoimmune process. The direct injury to the tubes affects the reabsorption process and the urine concentration capacity. Exposure to printing inks, paints, lacquers, varnishes, cleaning agents, petroleum products and glues showed high hazard ratios for end stage renal disease [12].

Several early biomarkers for CKD were proposed for the occupational exposed subjects, but there is no consensus on which would be the best for screening. The presence in urine of the kidney injury molecule type $1, \beta 2$-microglobulin, $\mathrm{N}$-acetyl- 
$\beta$-D-glucosamidase, cystatin $C[13,14]$ or of the enzymes from the lysis of the tubular cells (lactate dehydrogenase, $\gamma$-glutamyltransferase and albumin) [15] are used for research purposes and are not routinely performed.

Patients with T2D should avoid any exposure to kidney toxicants. Beside the cumulative effect, potentially additive mechanisms were described: glucose transporters (GLUT1, and GLUT5) in basolateral membrane of proximal renal tubules mediate the arsenic reuptake8. As the expression and the functionality of these transporters is increased in diabetes, the toxic effects of arsenic might be enhanced in T2D $[16,17]$.

\section{Clinical monitoring of type 2 diabetes patients with chronic kidney disease}

It is estimated that $40 \%$ of the patients with diabetes will develop chronic renal disease (CKD) [18]. The significance of the health impact of diabetes is also illustrated by the fact that more than $45 \%$ of patients having renal transplant or dialysis have diabetes as underlying cause for the renal failure [19]. Due to the long asymptomatic period, there is a shorter duration between the clinical diagnosis and the overt renal disease in T2D. After the initial diagnosis of nephropathy, most patients reach the end stage renal disease after 5-7 years [20].

Although microalbuminuria was long time considered the first sign of diabetic nephropathy [21], recent data tends to highlight other early diabetic kidney signs: the decline in the erythropoietin synthesis [22], the abnormal vitamin D metabolism or of the renin angiotensin axis $[23,24]$.

Current clinical diabetes guidelines recommend the measurement of the HbAlc every 3 months, the determination of albumin-to creatinine ratio (normal values $<30 \mathrm{mg} / \mathrm{g}$ creatinine) in the urine spot and the estimated glomerular filtration rate (eGFR) at least once a year $[25,26]$. The same guidelines underline the variability of the albumin-to creatinine ratio with exercise, infection, congestive heart failure, marked hyperglycaemia and hypertension and the necessity to exclude these possible influencers. A recommended approach is the determination of the albumin-to creatinine ratio in 2-3 specimens of urine in 3-6 month interval to establish a diagnosis of albuminuria. Concerning eGFR, a value of less than $60 \mathrm{ml} /$ $\min / 1.73 \mathrm{~m} 2$ should be considered abnormal. Since diabetic nephropathy is a microvascular complication, retinopathy is frequently present, however, for the kidney disease it has been proven to less sensitive in T2D than in T1D [27]. Albuminuria and/or an active urinary sediment (a sediment containing red cells, leucocytes, cellular casts) is currently considered as marker of kidney damage (classified as stage 1 or 2 of CKD, depending on the eGFR) and should be referred to a kidney specialist for diagnostic and evaluation of the risk factors for progressive disease even if eGFR is $\geq 90 \mathrm{ml} / \mathrm{min} / 1.73 \mathrm{~m} 2$. Risk factors for CKD progression are considered elevated blood pressure, glycaemia and albuminuria.

Other tests included in the yearly T2D evaluation are the lipid profile, liver tests, vitamin B12 in metformin treated patients, serum potassium in patients treated with ACE inhibitors, angiotensin receptor II blockers and diuretics [26]. It is foreseen that one or several more subtle parameters for glomerular filtration (urine transferrin, fibronectin, laminin, ceruloplasmin), podocyte injury (nephrin, podocalyxin, podocin), glomerulo-endothelial injury (glycoaminoglycan), tubular reabsorption, (cubilin, megalin, $\beta 2$ microglobulin, $\alpha 1$ microglobulin, retinol binding protein, cystatin $C$ ), tubular integrity (urine alkaline phosphatase, N-acetylglucosaminidase, alanin aminopeptidase, kidney injury molecule, type IV and type I collagen, matrix metalloproteinase 9), inflammation (PGE2 or PGF2 $\alpha$, $\mathrm{TNF} \alpha$, Interleukin 6 , resistin, interleukin $1 \alpha, \mathrm{MCP}-1$ among others), oxidative stress (8-oxo-7,8-dihydro2-deoxyguanosine), growth factors (transforming growth factor $\beta 1$, vascular endothelial growth factor A, connective tissue growth factor) $[24,28,29,30]$ will replace the current screening tests, as they are able to detect the kidney disease in earlier stages. Transferrin excretion is another candidate, as it was proven to proceed albuminuria in a larger cohort [31].

More recently, based on the characteristics that are generally available for screening (age, duration of diabetes, BMI, blood pressure, inflammatory markers, albumin, haemoglobin, and $\mathrm{HbA} 1 \mathrm{C}$ ) and the adding of a cytokine profile (monocyte chemoattractant protein-1, C-Chemokine ligand-18 and macrophage migration inhibitory factor), four phenotypes have been described: the cardiovascular phenotype (high blood pressure, high cholesterol); the microvascular complications with advanced diabetes related CKD one (the presence of retinopathy and a more advanced kidney disease); the advanced CKD with less anaemia, lower weight and $\mathrm{HbA1} \mathrm{c}$ and the hypercholesteraemic, younger, less severe CKD [32].

This phenotypical approach is not a novelty by itself, but has not been so extensively applied in T2D as it has been for asthma, for example. The future better 
characterization of T2D phenotypes might help to distinguish the lower from the higher risk patients for different occupational activities.

\section{Type 2 diabetes with chronic kidney disease evaluation in occupational medicine}

As mentioned above the renal nitrogen excretion is not necessarily as low as to impose dialysis in CKD associated with T2D and definitely not incompatible with an adjusted occupational activity in all cases. Therefore, adults with T2D and nephropathy should be the subject of a comprehensive examination by the occupational medicine practitioner.

High risk activities for accidents or consequences of an accident (working in possible contact with ionizing radiation, electromagnetic fields of $0-300$ $\mathrm{Hz}$, working at height or in an environment with high temperature, drivers), special working conditions (in isolated locations, in night shifts), activities requiring high physical demand (firefighter, rescue team worker) and exposure to potential nephrotoxic substances, are specifically required by our legislation to pass a thoroughly evaluation for fitness capacity. This evaluation implies an assessment of the stability of the diabetes, the frequency of the hypoglycaemic episodes, but also offers the possibility to inform the employer to provide the needed support for the proper management of the disease: access to regular meals, organization of the work flow (allowing, for example, breaks for insulin administration) etc.

Exposure to persistent organic pollutants (polychlorinated biphenyls, organochlorine pesticides, polycyclic aromatic hydrocarbons, phthalates etc) [6], heavy metals (arsenic, cadmium) [7] need to be avoided as they have a potential role in the pathogeny of diabetes. With correct technical measures, all employees should avoid occupational contact with these substances, not only by those already diagnosed with T2D.

A special concern of the occupational physician refers to the safety of patients with T2D and and that of their co-workers in the presence of hypoglycemia. There is no test available to identify the time a patient spent at lower glycaemic levels in the last months, as we have the HbA1C for the hyperglycaemic state. At the workplace, recurrent episodes need a combined effort of the diabetes specialist and of the occupational physician to identify what types of treatment may mitigate the risk and if there is no need for job change [25]. Due to the inconclusive benefits on complications incidence and risks of adverse effects of more aggressive therapy, including hypoglycemia, the latest recommendation of the American College of Physicians is to increase the target for the HbA1C in T2D to $7-8 \%$ and to modify the pharmacological treatment if $\mathrm{HbA} 1 \mathrm{C}$ is less than 6.5\% [33]. Therefore, the occupational medicine specialist should discuss interpretation of the $\mathrm{HbA} 1 \mathrm{C}$ with the treating physician.

In what concerns the work ability of the person with T2D, Weisman et al. [34] (have shown that the work characteristics and diabetes related factors almost equally contribute to the fatigue of the diabetic employee. In the regression model derived from the investigation of 292 persons with insulin dependent diabetes, $19.1 \%$ of the variance in fatigue was explained by work factors (lack of job support, the interaction between job demand and job control) and $29 \%$ was explained by the diabetes related factors (diabetes related symptoms, insulin regimen re-adjustment) [34]. If the diabetic employee allows disclosure of his medical condition, information on the management of this disease should be explained to the supervisor and to colleagues, to create the necessary support.

Given the complex interaction between work and diabetes, it is important to characterize the profile of the diabetic with nephropathy using the medical history, the clinical examination and the usual biological markers that are available to the occupational physician. Obesity, hypertension, anaemia, retinopathy and lack of diabetes control are more frequently found in the CKD nephropathy than in T2D without clinically manifest nephropathy.

Currently available biomarkers such as creatinine, urea, uric acid in serum, albumin/creatinine ratio in urine, eye fundus examination, should be performed at least annually or whenever decided by the occupational or by the treating physician. HbA1C should be measured every 3 months. The American Diabetes Association also recommends the following vaccines to be administered annually: influenza, 23-valent pneumococcal polysaccharide vaccine for persons $\leq 64$ years, and 3 dose series of hepatitis $B$ vaccines for persons with ages of 19-59 years. This recommendation is, however, supported by evidence from poorly controlled or uncontrolled studies [26].

\section{Conclusion}

Consequentially, a comprehensive investigation and close collaboration with the treating physician is required for each particular case. As there is 
evidence that reduction in blood pressure prevents kidney deterioration, attention should be focused on the reduction of blood pressure threshold to less than $140 / 90 \mathrm{mmHg}$, according to the patient's tolerance and the adherence to the renin-angiotensin blockade [35]. From the medical perspective, maintaining the therapeutic targets (blood pressure, albuminuria reduction, eGFR maintenance), prevention of complications and safety at work are the most important goals.

Detaille et al. [36] have explored the T2D patient's perspective and found that the most important needs were the acceptance of the disease and the ability to cope with it in the working environment. In this respect, finding proper disease management at work, including work adaptation and communication with managers and colleagues was emphasized. Patients also underlined their wish to receive sufficient information (including the financial implications of the treatment) and to have health professionals that can deal with the complications of T2D inside the company.

As part of the therapeutic team and in the current context of the increase in the prevalence of the T2D, the occupational physician has the difficult task to find solutions for the best support for these patients in the workplace. There are few guidelines available and maybe a local one, adapted to the Romanian working conditions and culture, should be of benefit for employees, employers and for the entire medical team. To substantiate an evidence-based guideline, in depth research of diabetes nephropathy phenotypes and finding reliable and affordable biomarkers are needed.

\section{References}

1. Mota M, Popa SG, Mota E, Mitrea A, Catrinoiu D, Cheta DM, Guja C, Hancu N, Ionescu-Tirgoviste C, Lichiardopol R, Mihai BM, Popa AR, Zetu C, Bala CG, Roman G, Serafinceanu C, Serban V, Timar R, Veresiu IA, Vlad AR. Prevalence of diabetes mellitus and prediabetes in the adult Romanian population: PREDATORR study. J Diabetes. 2016;8:336-44.

2. Sorrentino FS, Matteini S, Bonifazzi C, Sebastiani A, Parmeggiani F. Diabetic retinopathy and endothelin system: microangiopathy versus endothelial dysfunction. Eye. 2018; 32:1157-63.

3.Vinod PB. Pathophysiology of diabetic nephropathy. Clinical Queries: Nephrology. 2012;1:121-6.

4. Martínez-Castelao A, Navarro-González JF, Górriz JL, de Alvaro

F. The Concept and the Epidemiology of Diabetic Nephropathy Have Changed in Recent Years. J Clin Med. 2015;4:1207-16.

5. Piccoli GB, Grassi G, Cabiddu G, Nazha M, Roggero S, Capizzi I, De Pascale A, Priola AM, Di Vico C, Maxia S, Loi V, Asunis AM, Pani A, Veltri A. Diabetic Kidney Disease: A Syndrome Rather Than a Single Disease. Rev Diabet Stud. 2015;12:87-109.

6. Pizzorno J. Is the Diabetes Epidemic Primarily Due to Toxins?. Integr Med (Encinitas). 2016;15:8-17.
7. Edwards J, Ackerman C. A Review of Diabetes Mellitus and Exposure to the Environmental Toxicant Cadmium with an Emphasis on Likely Mechanisms of Action. Curr Diabetes Rev. 2016;12:252-8.

8. Orr SE, Bridges CC. Chronic Kidney Disease and Exposure to Nephrotoxic Metals. Int J Mol Sci. 2017;18:1039.

9. Ahamed M, Siddiqui MK.Low level lead exposure and oxidative stress: current opinions. Clin Chim Acta. 2007;383:57-64.

10. Burckhardt BC, Drinkuth B, Menzel C, Konig A, Steffgen J, Wright SH, Burckhardt G. The renal $\mathrm{Na}(+)$-dependent dicarboxylate transporter, NaDC-3, translocates dimethyl- and disulfhydrylcompounds and contributes to renal heavy metal detoxification. J. Am. Soc. Nephrol. 2002;13:2628-38.

11. Barbier O, Jacquillet G, Tauc M, Cougnon M, Poujeol P. Effect of heavy metals on, and handling by, the Kidney. Nephron Physiol 2005;99:105-10.

12. Jacob S, Héry M, Protois JC, Rossert J, Stengel B. New insight into solvent-related end-stage renal disease: occupations, products and types of solvents at risk. Occup Environ Med. 2007;64:843-8.

13. Prozialeck WC, Edwards JR. Early biomarkers of cadmium exposure and nephrotoxicity. Biometals. 2010;23:793-809.

14. Chen J, Li M, Lv Q, Chen G, Li Y, Li S, Mo Y, Ou S, Yuan Z, Huang $\mathrm{M}$, Jiang Y. Blood lead level and its relationship to essential elements in preschool children from Nanning, China. J. Trace Elem. Med. Biol. 2015;30:137-141.

15. Jan AT, Azam M, Siddiqui K, Ali A, Choi I, Haq QM. Heavy Metals and Human Health: Mechanistic Insight into Toxicity and Counter Defense System of Antioxidants. Int J Mol Sci. 2015;16:2959229630.

16. Szablewski L. Distribution of glucose transporters in renal diseases. J Biomed Sci. 2017;24:64.

17. Kataria A, Trasande L, Trachtman H. The effects of environmental chemicals on renal function. Nat Rev Nephrol. 2015;11:610-25.

18. Gheith O, Farouk N, Nampoory N, Halim MA, Al-Otaibi T. Diabetic kidney disease: world wide difference of prevalence and risk factors. J Nephropharmacol. 2015;5:49-56.

19. J. Jameson and L. Loscalzo, Harrison's Nephrology and Acid-Base Disorders, New York: McGraw-Hill Medical, 2017.

20. Persson F, Rossing P. Diagnosis of diabetic kidney disease: state of the art and future perspective. Kidney Int Suppl 2011;8:2-7.

21. Viberti GC, Hill RD, Jarrett RJ, Argyropoulos A, Mahmud U, Keen $\mathrm{H}$. Microalbuminuria as a predictor of clinical nephropathy in insulindependent diabetes mellitus. Lancet. 1982;1:1430-2.

22. Bosman DR, Winkler AS, Marsden JT, Macdougall IC, Watkins PJ. Anemia with erythropoietin deficiency occurs early in diabetic nephropathy. Diabetes Care 2001;24:495-9.

23. Chokhandre MK, Mahmoud MI, Hakami T, Jafer M, Inamdar AS. Vitamin D \& its analogues in type 2 diabetic nephropathy: a systematic review. J Diabetes Metab Disord. 2015;14:58.

24. LeeSY, Choi ME. Urinarybiomarkers for early diabeticnephropathy: beyond albuminuria.Pediatr Nephrol.2015;30:1063-75.

25. American Diabetes Association. Diabetes and Employment. Diabetes Care. 2010;33 (Suppl1): S82-S86.

26. American Diabetes Association. Comprehensive Medical Evaluation and Assessment of Comorbidities: Standards of Medical Care in Diabetes-2019. Diabetes Care 2019 42(Suppl 1): S34-S45. https://care.diabetesjournals.org/content/42/Supplement_1/S4 27. He F, Xia X, Wu XF, Yu XQ, Huang FX. Diabetic retinopathy in predicting diabetic nephropathy in patients with type 2 diabetes and renal disease: a meta-analysis. Diabetologia 2013;56:457-66.

28. Uwaezuoke SN.The role of novel biomarkers in predicting diabetic nephropathy: a review. Int J Nephrol Renovasc Dis 2017:10: 221-31. 29. Li Y, Xia W, Zhao F, Wen Z, Zhang A, Huang S, Jia Z, Zhang Y. Prostaglandins in the pathogenesis of kidney diseases. Oncotarget. 2018;9:26586-26602

30. Al-Rubeaan K, Siddiqui K, Al-Ghonaim MA, Youssef AM, AlSharqawi AH, AlNaqeb D. Assessment of the diagnostic value of different biomarkers in relation to various stages of diabetic nephropathy in type 2 diabetic patients. Sci Rep. 2017;7:2684.

31. Kazumi T, Hozumi T, Ishida Y, Ikeda Y, Kishi K, Hayakawa 
M, Yoshino G.Increased urinary transferrin excretion predicts microalbuminuria in patients with type 2 diabetes. Diabetes Care. 1999;22:1176-80.

32. Montero RM, Herath A, Qureshi A, Esfandiari E, Pusey CD, Frankel AH, Tam FWK. Defining Phenotypes in Diabetic Nephropathy: a novel approach using a cross-sectional analysis of a single centre cohort. Sci Rep. 2018;8:53.

33. Qaseem A, Wilt TJ, Kansagara D, Horwitch C, Barry MJ, Forciea MA, Clinical Guidelines Committee of the American College of Physicians. Hemoglobin A1c targets for glycemic control with pharmacologic therapy for nonpregnant adults with type 2 diabetes mellitus: a guidance statement update from the American College of Physicians. Ann Intern Med 2018; 168: 569-76.

34. Weijman I, Ros WJ, Rutten GE, Schaufeli WB, Schabracq MJ, Winnubst JA. Fatigue in employees with diabetes: its relation with work characteristics and diabetes related burden. Occup Environ Med. 2003;60 Suppl 1:i93-i98.

35. Umanath K, Lewis JB. Update on Diabetic Nephropathy: Core Curriculum. Am J Kidney Dis. 2018; 71: 884-95.

36. Detaille SI, Haafkens JA, Hoekstra JB, van Dijk FJH. What employees with diabetes mellitus need to cope at work: Views of employees and health professionals. Patient Education and 